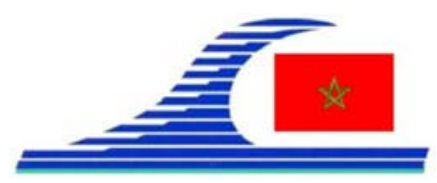

Conférence Méditerranéenne Côtière et Maritime

EDITION 2, TANGER, MAROC (2011)

Coastal and Maritime Mediterranean Conference

Disponible en ligne - http://www.paralia.fr - Available online

\title{
Analyses des eaux portuaires. Utilisation de la technique des biopuces au Port de Beaulieu (Alpes-Maritimes)
}

\section{Pierre DOGLIOLI $^{1}$, Pierre ESCOUBET ${ }^{1}$, Yves PRÜFER ${ }^{2}$, Sophie PAGNOTA ${ }^{3}$, Arnaud TANGUY ${ }^{4}$, Philippe PISSARELLO ${ }^{5}$, Catherine MARTIN ${ }^{6}$}

1. Plate-forme Technologique Biopuces, 11, rue Lamartine, 06000 Nice, France. pierredoglioli@wanadoo.fr

2. Laboratoire de l'Environnement Durable, Nice, France.

3. Centre Commun de Microscopie Electronique, Nice, France.

4. Station Biologique UMR CNRS 7 144, Roscoff, France.

5. Nice Côte d'Azur, Nice, France.

6. Port de Beaulieu - Plaisance, Beaulieu sur mer, France.

\section{Résumé :}

Les eaux portuaires contiennent des quantités variables, selon l'activité du port, de xénobiotiques tels que les métaux lourds, les hydrocarbures, les PCB, les pesticides et d'autres produits. La majorité de ces substances sont toxiques pour les organismes biologiques qui résident à leur contact de manière permanente (coquillages, crustacés, algues) ou de manière passagère (poissons, mollusques).

Nous avons voulu mesurer les impacts de l'ensemble de ces substances sur le génome des animaux en utilisant comme modèle, la moule de Méditerranée et comme technique d'analyses la technique des biopuces que nous avons couplé à un ensemble de dosages chimiques et à une étude de microscopie électronique.

Les premiers résultats obtenus valident les hypothèses que nous avions envisagé dès le départ, c'est-à-dire, des interactions très fortes sur ces génomes.

Comme les eaux portuaires ne sont par limitées à l'espace du port mais circulent, et souvent en direction de plages utilisées par des baigneurs, il serait très utile, dans un deuxième temps, en utilisant les mêmes techniques, d'analyser les eaux de baignades.

Mots Clés : Eaux portuaires - Xénobiotiques - Stations artificielles de moules Biopuces - Microscopie Electronique - Analyse des génomes

\section{Introduction}

Les ports sont par définition des zones marines prévues et équipées, pour recevoir des activités humaines basées sur la circulation et l'accostage de navires et bateaux de tailles variables. Pour répondre à une demande économique, en particulier touristique, de plus en plus forte, les communes ont dû mettre en place et construire de nombreux sites portuaires dans le but d'accueillir l'ensemble des bateaux et, de développer les activités économiques concernant le transport des marchandises. A titre d'exemple il existe plus de 136 ports en région Provence-Alpes-Côte d'Azur. 
La connaissance de la Mer :

un vecteur du développement durable en Méditerranée

Au cours du temps, l'évolution de la demande a entrâné les ports à accueillir de plus en plus d'unités de tailles sans cesse grandissantes et des activités commerciales, en particulier le transport des touristes, n'ont fait que croître.

Toute cette activité a entraîné, inévitablement, une concentration de produits de nature chimiques qui au cours du temps se sont déposés au niveau de la vase et pour une grande partie est restée, selon ses propriétés de solubilités respectives, en suspension dans les eaux portuaires. Comme les ports sont des systèmes relativement fermés aux influences extérieures (courants, marées) il en résulte que ces accumulations chimiques sont de nature à développer des phénomènes de toxicité directement au niveau des organismes biologiques qui y résident ou qui circulent fréquemment dans les ports constituant de fait des espèces sensibles et plus loin au niveau de la chaîne alimentaire des proies ingérées par de plus gros organismes (RAMADE, 1997).

Pour connaître les impacts réels de cet ensemble chimique constitué par les métaux lourds, les hydrocarbures et les PCB essentiellement, il nous fallait utiliser une méthode qui nous permette d'analyser le génotype et non le phénotype, ce qui était fait jusqu'à présent, nous permettant ainsi de mesurer sur la molécule d'ADN de chacun des chromosomes, les sur- expressions, les inhibitions et voire les mutations subies chez l'animal dans ce contexte pollué.

Nous avons mis au point une technique basée sur les Biopuces qui permet cette analyse de façon approfondie. Pour apporter des informations supplémentaires nous avons par microscopie électronique, à balayage et transmission, essayé de repérer la localisation intra cellulaire des polluants. Ce que nous avons réussi à visualiser.

Notre travail qui a porté essentiellement sur un modèle, la moule de Méditerranée, peut très bien s'appliquer à d'autres modèles animaux (planctons).

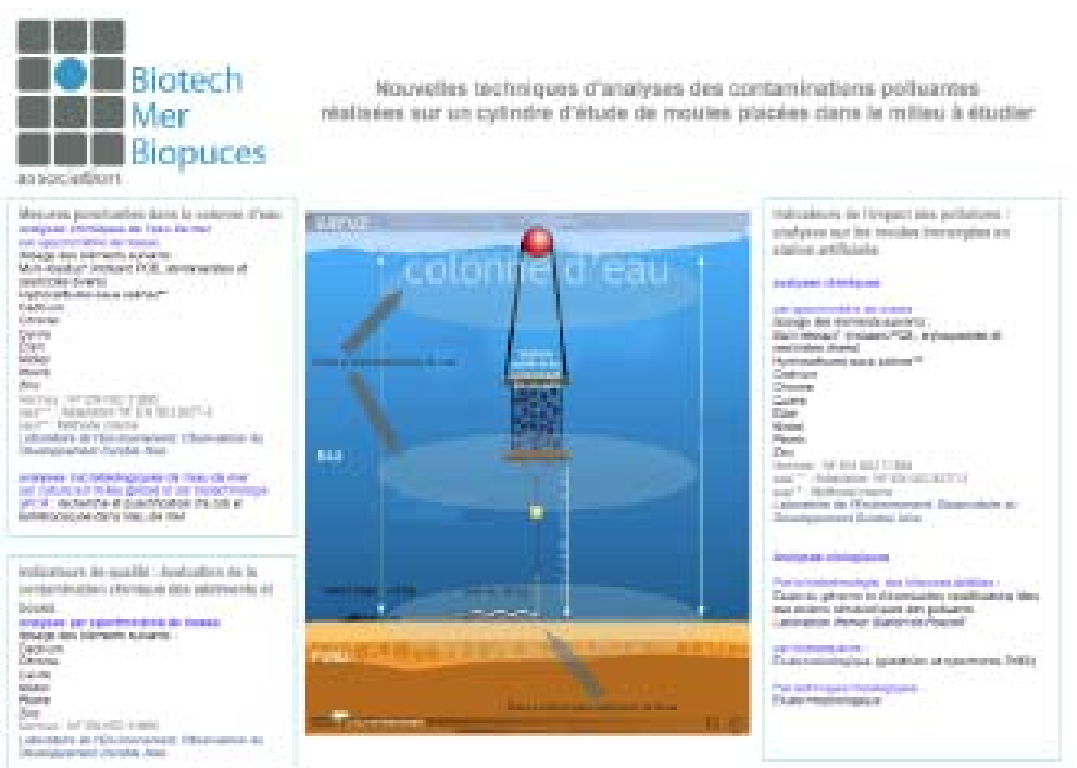

Figure 1.Schéma de notre modèle d'étude. 


\section{Matériel et méthodes}

\subsection{Technique générale utilisée}

Nous avons placé à 6 endroits définis par une spécificité particulière (zone de carénage, zone d'arrivée d'un cours d'eau, zone de stationnement de grandes unités...) des stations artificielles de moules (ANDRAL, 1997 ; ANDRAL \& STANISIERE, 1999) Pour chaque zone ainsi définie nous avons :

- dosé les métaux lourds et les hydrocarbures des eaux portuaires

- analysé les bactéries présentes (Escherichia coli et entérocoques)

- dosé les métaux lourds dans les sédiments

- dosé les métaux lourds dans les moules après 2 mois d'exposition

- réalisé les biopuces sur les moules du site après 2 mois d'exposition

- réalisé les microscopies électroniques sur les branchies des moules après 2 mois d'exposition.

\subsection{Matériel}

a-Station artificielle de moules: moules de Méditerranée, Mytilus galloprovincialis (COSSA et al., 1998) 2 kilos par cage. Prélevées à partir d'une ferme aquacole située à $30 \mathrm{~km}$ de la zone portuaire étudiée.

b-Analyses chimiques des eaux, des sédiments et des moules : réalisées au laboratoire du Développement Durable - Nice (SCHREIER 1984; IFREMER, 1998 ; BERGMAN, 1993).

c- Analyses bactériologiques : en microplaques sur milieu fluorescent permettant de détecter l'activité enzymatique de la béta glucuronidase d'Escherichia coli et la béta galactosidase des entérocoques - Laboratoire du développement Durable - Nice.

d-Microscopie électronique : les branchies des moules ont été fixées dans le mélange eau de mer artificielle - 2\% glutaraldéhyde - Centre Commun de Microscopie Electronique - Nice.

e- Biopuces : les moules entières sont plongées dans l'azote liquide et conservées dans la carboglace avant expédition à la Station de Biologie Marine - Roscoff.

\subsection{Méthodes}

Les moules utilisées, Mytilus galloprovincialis, ont été prélevées sur les bordures latérales d'une ferme aquacole située à $30 \mathrm{~km}$ de la zone portuaire étudiée. Elles ont été calibrées à la taille de $4 \mathrm{~cm}$ pour avoir un échantillonnage homogène. Chacune des cages à moules reçoit 100 moules. Chaque station est immergée dans un site déterminée par une caractéristique soit géographique (rivière) soit économique (carénage, bateaux de pêche, ...). Les cages se trouvaient à $50 \mathrm{~cm}$ du fond et maintenues en surface par un flotteur. 
La connaissance de la Mer :

un vecteur du développement durable en Méditerranée

a Dosages chimiques des métaux lourds : 6 métaux ont été sélectionnés pour cette étude (Cadmium, Chrome, Cuivre, Nickel, Plomb, Zinc). Après minéralisation des échantillons et dosage par ICP/AES (NF EN ISO 11885, AUBERT et al., 1979).

b Dosages bactériologiques : 2 types bactériens ont été choisis (entérocoques et Escherichia coli) - Techniques des microplaques avec révélateur fluorescent spécifique pour chaque type bactérien. Les prélèvements ont été effectués de manière stérile dans des flacons de PET stérilisés à l'autoclave.

c Microscopie électronique à balayage et à transmission : les branchies de 2 moules / lot ont été fixées dans le mélange eau de mer artificielle - $2 \%$ glutaraldéhyde pendant $24 \mathrm{~h}$ à $+4^{\circ} \mathrm{C}$. Elles ont été incluses (colloïdine) et coupées au microtome à congélation. Epaisseur des coupes : 5 microns

Microscope électronique marque Philips. Grossissement : 10000

d Biopuces : les moules entières, 20, sont plongées dans de l'azote liquide et transférées dans de la carboglace pour être expédiées au laboratoire de traitement. Elles ont subi sur place les traitements suivants :

- Extraction des ARN totaux

- Synthèse des sondes marquées (CYT3 et CYT 5)

- Hybridation des sondes marquées

- Analyse des données à l'aide du logiciel GenePix

\section{Résultats}

a Chimie des eaux, sédiments et moules: présence des métaux lourds en quantité variable :

- Taux élevés de Cuivre et de Zinc dans les eaux : respectivement 20 et 32 microgrammes /L

- Taux élevés de Cuivre, Zinc et Plomb dans les sédiments : 400 mg/kg - 780 mg/kg

$-500 \mathrm{mg} / \mathrm{kg}$

- Taux élevés de Cuivre et de Chrome dans les moules : $700 \mathrm{mg} / \mathrm{kg}$ et $580 \mathrm{mg} / \mathrm{kg}$ (D’SILVIAC-KUREISHY, 1978 ; ASSO, 1985)

b Hydrocarbures : teneur moyenne des eaux 70 microgramme/L

c Bactériologie :

- Escherichia coli : en moyenne des valeurs inférieures aux normes acceptées (moins de 2000/ $100 \mathrm{ml}$ )

- Entérocoques : Très loin des 10000 / 100 ml (en moyenne 1500 /100 ml)

d Microscopie électronique :

- balayage : visualisation de zones où les cils ont disparu complètement

- transmission : visualisation d'inclusions de nature métallique dans les cellules des branchies de la moule dans toutes les zones du port

e Biopuces : sur 8000 gènes significatifs étudiés 800 , soit $10 \%$ du total présentent des modifications intéressantes et significatives (LE MEUR et al., 2004) : 
- 508 gènes présentent une inhibition en général des activités sexuelles dans la formation des gamètes et aussi une diminution des activités métaboliques en particulier lors de la synthèse des protéines.

- 270 gènes présentent une surexpression significative dans les sites des défenses immunitaires, la respiration cellulaire et le stress oxydatif.

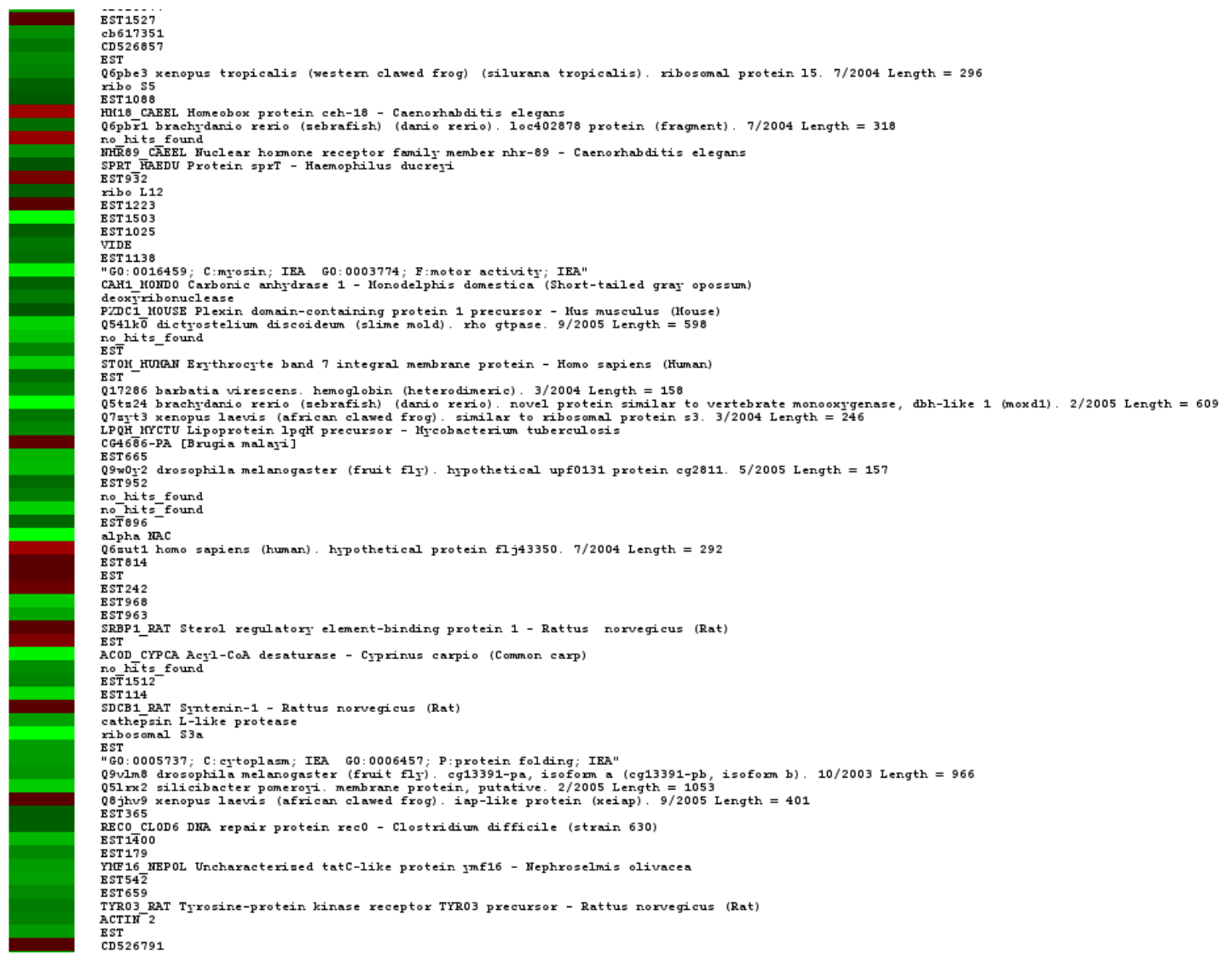

Figure 2. Résultats obtenus à partir du logiciel Gen Pix et traduisant des inhibitions(en vert) et des surexpressions (en rouge)- Fragment de résultats.

\section{Conclusions}

Cette étude rassemblant une association de plusieurs techniques, mais dont l'essentielle reste celle des biopuces, a montré :

- la présence importante des métaux lourds aussi bien dans les eaux, les sédiments et les organismes vivants placés dans ces zones portuaires,

- la présence plus ou moins importante de contaminations bactériennes limitées pour la période dans laquelle nous avons travaillé (14 octobre 2010 - 15 décembre 2010 période froide),

- les impacts sur le génome de ces organismes traduisant une atteinte des structures génétiques de l'ADN,

- la concentration des particules métalliques et autres dans les branchies des moules. 
La connaissance de la Mer :

un vecteur du développement durable en Méditerranée

Elle est donc innovante par la mise en évidence de ces atteintes sur les espèces vivantes placées au contact, pour une durée limitée, des eaux portuaires.

En juin 2011 nous démarrons, dans les mêmes conditions une deuxième campagne pour valider la première et voir les variantes pouvant apparaître en période où les eaux sont plus chaudes.

Remerciements : Le projet a été financé par la Direction Régionale de la Recherche et de la Technologie - PACA et par la Société Victory Technologie - Nice.

\section{Références bibliographiques}

ANDRAL B. (1997). Réseau Intégrateurs Biologiques : étude de la contamination chimique du milieu littoral méditerranéen. Rapport de contrat dans le cadre de la convention n 95095 pour l'Agence de l'Eau RMC, Ifremer Toulon.

ANDRAL B., STANISIERE J.Y. (1999). Réseau Intégrateurs Biologiques : évaluation de la qualité des eaux basée sur l'utilisation de stations artificielles de moules en Méditerranée. Rapport de contrat dans le cadre des conventions Nº 991452 et 992461 pour l'Agence de l'Eau RMC, Ifremer Toulon.

ASSO A. (1985). Etude des teneurs globales en métaux lourds chez la moule Perna perna(L) dans la région d’Alger. Journ. Etud. Pollut. CIESM, 6, pp 385-394.

AUBERT M., REVILLON P., LAUMOND F., AUBERT J. (1979). Métaux lourds en Méditerranée. Campagnes Océanographiques du CERBOM, 1965-1979. Rev. Int. Ocean. Medic. Vol. 56-57, 304 p.

BERGMAN A. (1993). Concentration of PAH, PCBs and heavy metals in the blue mussel Mytilus edulis. 1993 Project Polwad/Beon Effekt Ministry of Transport, Public Work and Water Managment of Netherlands.

COSSA D., ABARNOU A., ANDRAL B., BOUGRIER S., BUESTEL D., CLAISSE D., HATT P.J., STANISIERE J.Y. (1998). De l'usage des moules pour le suivi de la contamination chimique le long des côtes méditerranéennes françaises. Rapport Ifremer DEL/PC, RA 708. Mars 1998, 76 p.

D'SILVIAC-KUREISHY W. (1978). Experimental studies of the accumulation cooper and zinc in green mussel. Mar. Poll. Bull. 9, pp 187-190.doi:10.1016/0025-326X(78)90176-5 IFREMER (1998). Les contaminants chimiques dans les sédiments du littoral méditerranéen. IFREMER. Surveillance du milieu marin-Travaux RNO.

LE MEUR N., LAMIRAULT G., BILHOUEE A., LEGER, J.J. (2004). A dynamic, web-accessible resource to process raw microarray scan data into consolidated gene expression values. Importance of replication. Nucleic Acids Research, 32 (18), pp 5349-5358. doi:10.1093/nar/gkh870

RAMADE F. (1997). Ecotoxicologie. Masson $2^{\mathrm{e}}$ Edition Paris, 228 p.

SCHREIER H. (1984). Asbestos fibres and associated trace metal toxicity in stream water. 11th Annual Aquatic Toxicity Workshop. Richmond (BC) Nov. 13-15, Canada. 